\title{
Plasma Stability in a Tokamak with Reactor Technologies Taking into Account the Pressure Pedestal
}

\author{
S. Yu. Medvedev ${ }^{a, b, c, *}$, A. A. Martynov ${ }^{a, b, c, * *}$, S. V. Konovalov ${ }^{b, c, * * *}$, V. M. Leonov ${ }^{b, c, * * * *}$, \\ V. E. Lukash ${ }^{b, c, * * * * *}$, and R. R. Khayrutdinov ${ }^{b, c, * * * * * *}$ \\ ${ }^{a}$ Keldysh Institute of Applied Mathematics, Russian Academy of Sciences, Moscow, 125047 Russia \\ ${ }^{b}$ National Research Center Kurchatov Institute, Moscow, 123182 Russia \\ ${ }^{c}$ Institution Project Center ITER, Rosatom, Moscow, 123182 Russia \\ *e-mail: medvedevsyu@keldysh.ru \\ **e-mail:martynov@keldysh.ru \\ ***e-mail: konovalov_sv@nrcki.ru \\ ****e-mail: leonov_vm@nrcki.ru \\ *****e-mail: lukasho8@yandex.ru \\ ******e-mail:khayrutd@mail.ru
}

Received May 10, 2021; revised May 30, 2021; accepted June 2, 2021

\begin{abstract}
Studying stationary regimes with high plasma confinement in a tokamak with reactor technologies (TRT) [1] involves calculating the plasma stability taking into account the influence of the current density profiles and pressure gradient in the pedestal near the boundary. At the same time, the operating limits should be determined by the parameters of the pedestal, which, in particular, are set by the stability limit of the peeling-ballooning modes that trigger the peripheral disruption of edge localized modes (ELM). Using simulation of the quasi-equilibrium evolution of the plasma by the ASTRA and DINA codes, as well as a simulator of magnetohydrodynamic (MHD) modes localized at the boundary of the plasma torus based on the KINX code, stability calculations are performed for different plasma scenarios in the TRT with varying plasma density and temperature profiles, as well as the corresponding bootstrap current density in the pedestal region. At the same time, experimental scalings for the width of the pedestal are used. The obtained pressure values are below the limits for an ITER-like plasma due to the lower triangularity and higher aspect ratio of TRT plasma. For the same reason, the reversal of magnetic field shear in the pedestal occurs at a lower current density, which causes the instability of modes with low toroidal wave numbers and reduces the effect of diamagnetic stabilization.
\end{abstract}

Keywords: tokamak, MHD stability, pressure pedestal

DOI: $10.1134 / \mathrm{S} 1063780 \mathrm{X} 21110222$

\section{INTRODUCTION}

The $\mathrm{H}$-mode, the regime with a high energy-confinement time in tokamak plasma, is accompanied by the formation of a transport barrier in the outer region of the plasma near the separatrix: improvement in confinement is associated with the pedestal height, i.e., the amount of pressure at the boundary of the transport barrier, which is an area with reduced transport coefficients. Achieving stationary $\mathrm{H}$-mode is one of the goals of the TRT project. At the same time, the operating limits of the installation are determined by stable plasma confinement with rather high values of normalized $\beta_{N}$ and the pressure on the pedestal. Limitations on the height of the pedestal are based on the assumption that the peeling-ballooning modes, localized at the plasma boundary, are the triggering mechanism for the development of ELM. The peeling-bal- looning modes are ideal magnetohydrodynamic (MHD) instabilities caused by large pressure gradients and the corresponding bootstrap current in the region of the transport barrier near the plasma boundary. The stability limits of such modes in the "pressure gradient-current density" plane greatly depend on the shape of the plasma, and the trajectory in this plane, along which the pedestal parameters evolve, depends on the plasma collisionality parameter $v^{*}[2,3]$. For an ITER-like plasma, at high values of $v^{*}$, bootstrapcurrent generation becomes less efficient, and ballooning modes with relatively large wave numbers $n>10$ mainly limit the plasma parameters. At low $\mathrm{v}^{*}$, modes with smaller $n=1-5$, which are destabilized by a large current density, are the most unstable, also due to reversal of the shear of the equilibrium magneticfield lines. Despite localization in the pedestal, the 
(a)

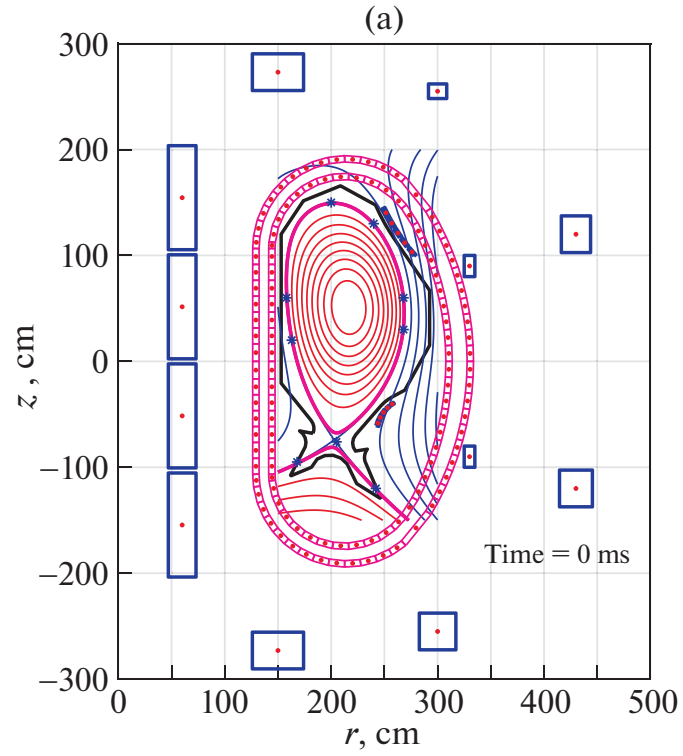

(b)

(c)
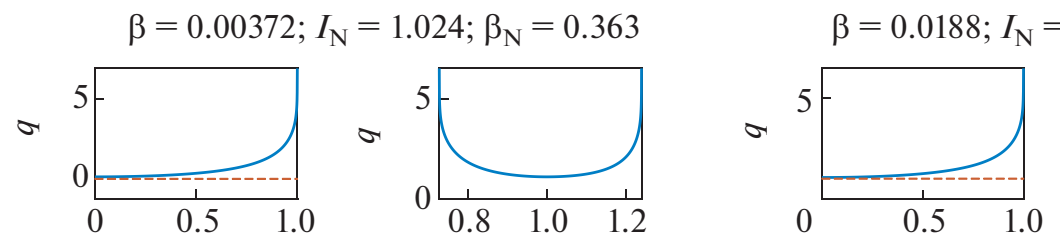

$1.03 ; \beta_{\mathrm{N}}=1.824$
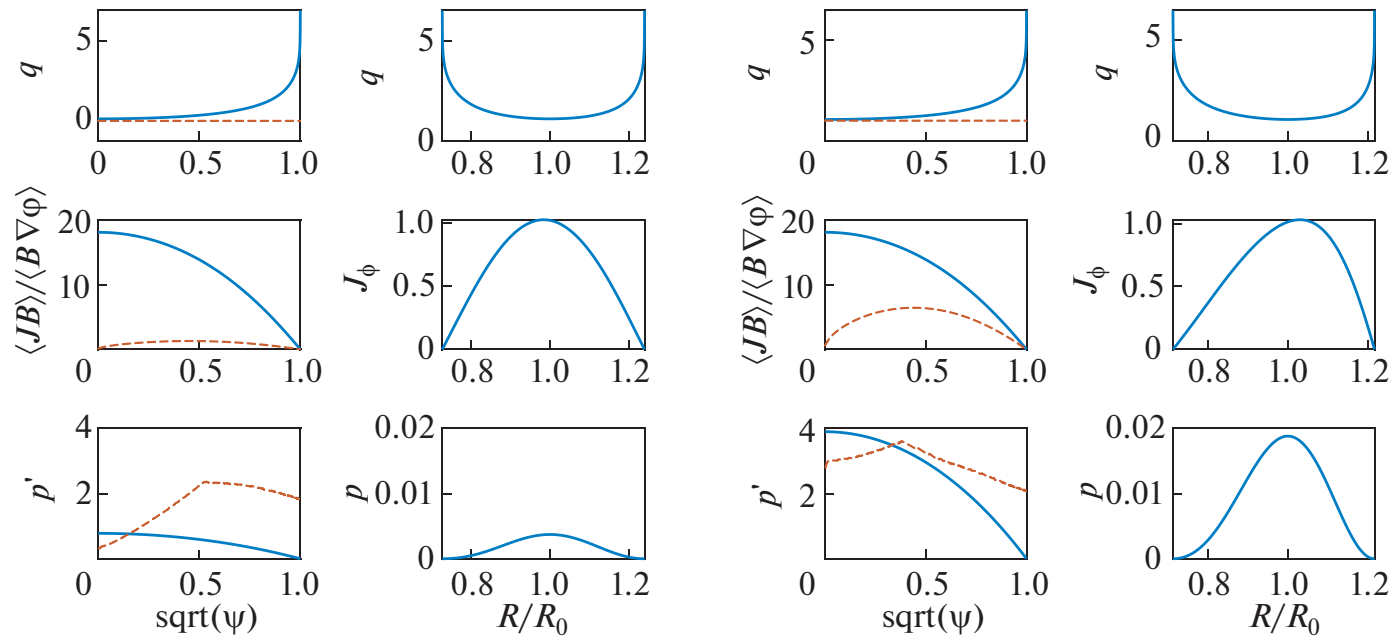

Fig. 1. TRT equilibrium with a free boundary: (a) the level lines of the poloidal flux function $\psi$. Plasma profiles for reference equilibria: (b) initial profiles, $\beta_{p}=0.16$; and (c) plasma pressure increased 5 times, $\beta_{p}=0.81$. The density of the collisionless bootstrap current and the limiting pressure gradient for ballooning modes are shown by dashed lines in the graphs for the longitudinal current density and pressure gradient, respectively.

peeling-ballooning modes have a complex spatial structure and numerical calculations using twodimensional codes are necessary to determine their stability. In calculations using the KINX code [4], the plasma extends up to the magnetic-field separatrix.

In Section 2, the reference equilibrium configurations of TRT are described and the stability limits against the external kink modes with increasing pressure are found. Section 3 describes the pedestal model, its application to the reference equilibria and the scaling for the width and height of the pedestal. Calculations of the maximum stability parameters of the pedestal, taking into account diamagnetic stabilization, are presented in Section 4. Finally, conclusions are made about the operating limits of TRT plasma.

\section{REFERENCE EQUILIBRIUM CONFIGURATIONS AND STABILITY LIMITS}

The equilibrium with a free boundary, calculated by the DINA code, sets the geometry of the plasma for studying the stability limits of the TRT (Fig. 1a). The plasma parameters correspond to a large plasma current $I_{\mathrm{p}}=4.8 \mathrm{MA}$ (toroidal field $B_{0}=8 \mathrm{~T}$ ), but a low 


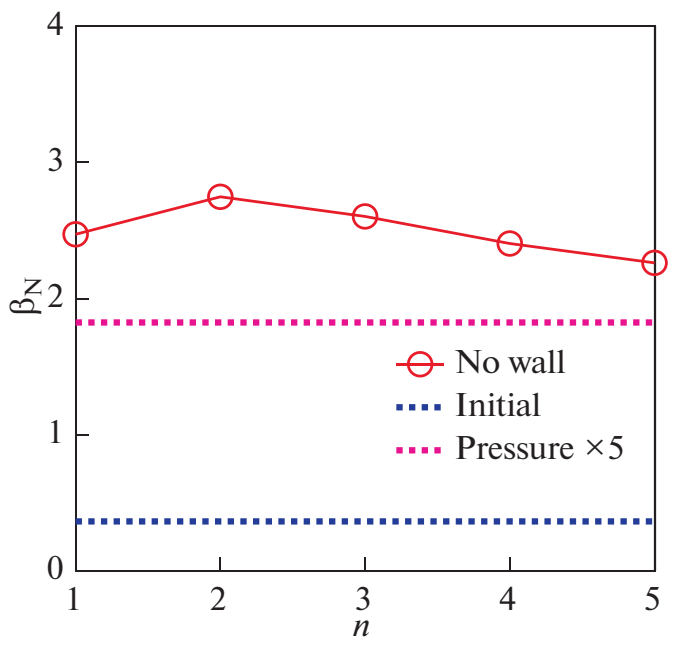

Fig. 2. Limiting values of the normalized beta for the stability of external kink modes with toroidal wave numbers $n=1-5$. The horizontal dotted lines show the values of $\beta_{N}$ for the equilibria from Fig. 1.

pressure (Fig. 1b). For a given separatrix coinciding with the plasma boundary, while conserving the current density profile $\langle\mathbf{j} \cdot \mathbf{B}\rangle_{\psi} /\langle\mathbf{B} \cdot \nabla \varphi\rangle_{\psi}$ parallel to the full helical equilibrium magnetic field $\mathbf{B}$ with volume averaging \langle\rangle$_{\psi}$ between the magnetic surfaces, and proportionally increasing the pressure, it is possible to obtain a sequence of equilibria for studying the plasma parameters limiting the MHD stability (Fig. 1c).

(a)
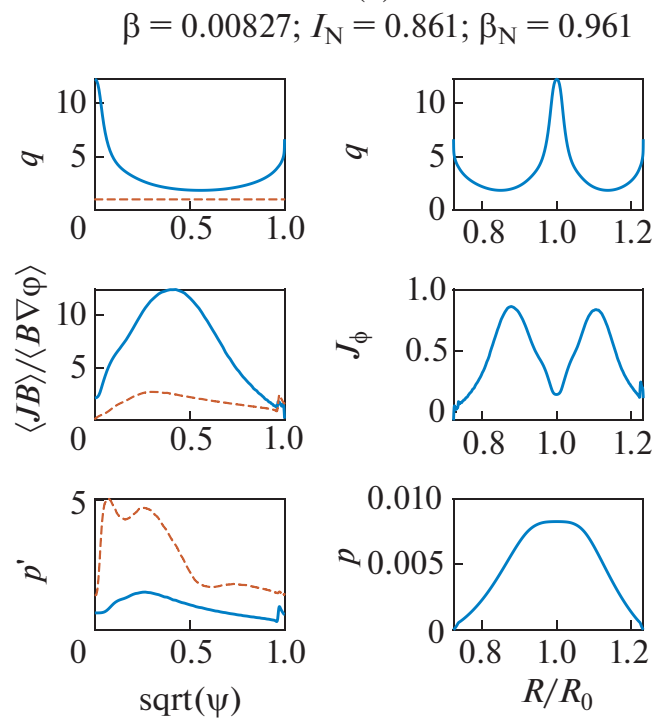

A natural condition for the operation of a tokamak in the stationary regime is the stability reserve with respect to large-scale external kink modes, which are responsible for the Troyon limit. Figure 2 shows the stability limits in units of normalized beta $\beta_{\mathrm{N}}$ for the modes with toroidal wave numbers $n=1-5 ; \beta_{\mathrm{N}}=\beta / I_{\mathrm{N}}$, $\beta=2 \mu_{0}\langle p\rangle_{\mathrm{V}} / B_{0}^{2}, \quad I_{\mathrm{N}}=I_{\mathrm{p}}[\mathrm{MA}] /\left(a[\mathrm{~m}] B_{0}[\mathrm{~T}]\right), \quad$ where $\langle p\rangle_{\mathrm{V}}, B_{0}, a$ are the pressure averaged over the volume of the plasma, the vacuum toroidal field at the geometric center, and the small radius of the plasma, respectively. We note that at higher values of $n$, diamagnetic stabilization may be significant [5]. Thus, equilibrium with $\beta_{\mathrm{N}}=1.8$ (Fig. 1c), versus limiting $\beta_{\mathrm{N}}<2.2$, is a conservative choice for the reference equilibrium with a peaking pressure profile $p_{0} /\langle p\rangle_{\mathrm{V}}=$ 3.4; the internal inductance of the equilibrium current density is $l_{i}(3)=0.74$.

Another variant of the equilibrium TRT configuration corresponds to the stationary regime obtained using the ASTRA code (Fig. 3) with a plasma current of $4 \mathrm{MA}$. In this case, due to noninductive current drive, the safety factor profile is nonmonotonic $\left(q_{\min }=1.85\right)$, while the pressure peaking factor is significantly lower $p_{0} /\langle p\rangle_{\mathrm{V}}=2.4$; the internal inductance is $l_{i}(3)=0.68$ (Fig. 3a). For the same plasma boundary, the limiting $\beta_{\mathrm{N}} \approx 2$ with respect to the stability of the external kink mode $n=1$ is lower (Fig. 3b) compared to $\beta_{\mathrm{N}} \approx 2.5$ for the first equilibrium (Fig. 2). Taking into account the stabilizing effect of a conducting wall, conformal to the plasma boundary

(b)

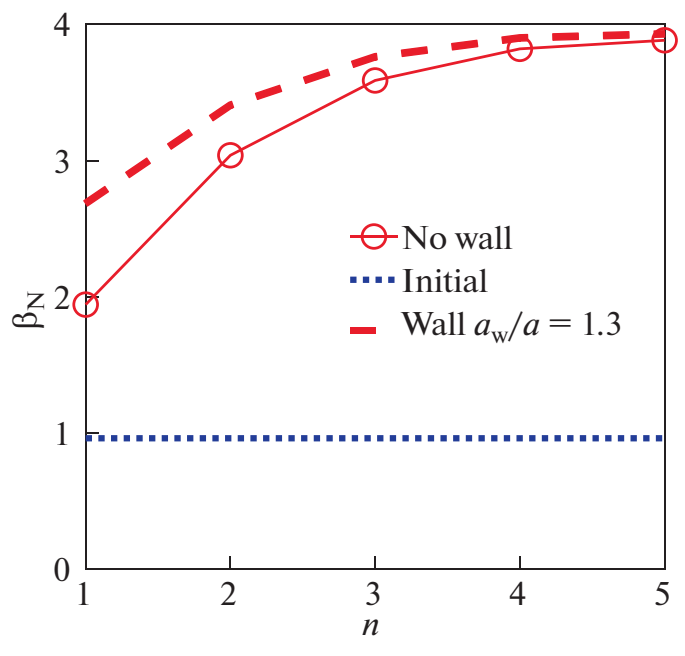

Fig. 3. (a) Plasma profiles for the reference equilibrium $\beta_{p}=0.55$ and (b) limiting values of the normalized beta for the stability of external kink modes with toroidal wave numbers $n=1-5$. The horizontal dotted line shows the value of $\beta_{\mathrm{N}}$ for the reference equilibrium. 
with a coefficient of 1.3 , makes it possible to increase this limit to 2.7, provided that the $n=1$ resistive wall mode (RWM), which remains unstable due to the finite conductivity of the wall, is stabilized.

It should be noted that the pressure limits are related to the plasma cross-section shape factor, which can be estimated as the product $q_{95} I_{\mathrm{N}}$, where $q_{95}$ is the value of the safety factor on a magnetic surface with a 95\% poloidal flux fraction inside the separatrix. This factor turns out to be less for TRT plasma with an aspect ratio of $A=2.15 / 0.56=3.8$, an elongation of $\kappa=2$ and a triangularity of $\delta=0.2: q_{95} I_{\mathrm{N}}=3.7-3.9$ compared to $q_{95} I_{\mathrm{N}}=4.4-5$ for ITER $(A=6.2 / 2=3.1$, $\kappa=1.8, \delta=0.4)$. Most likely, a lower shape factor is the reason for lowering the Troyon limit compared to $\beta_{\mathrm{N}}>3$ in similar ITER scenarios [5]. The limiting beta can be increased with a larger triangularity, as well as by optimizing the current and pressure profiles.

\section{PEDESTAL MODEL}

For self-consistent calculation of the bootstrap current taking into account collisionality, the density and temperature profiles are needed [2, 3]. The EPED1 code [6] uses the following parameterization in the pedestal for the electron density and temperature profiles:

$$
\begin{gathered}
n_{e}=n_{\text {sep }}+a_{n}\left\{\tanh \left[2\left(1-\psi_{\text {mid }}\right) / \Delta\right]\right. \\
\left.-\tanh \left[2\left(\bar{\psi}-\psi_{\text {mid }}\right) / \Delta\right]\right\} \\
T_{e}=T_{\text {sep }}+a_{T}\left\{\tanh \left[2\left(1-\psi_{\text {mid }}\right) / \Delta\right]\right. \\
\left.-\tanh \left[2\left(\bar{\psi}-\psi_{\text {mid }}\right) / \Delta\right]\right\},
\end{gathered}
$$

where $\Delta$ is the pedestal width, $\psi_{\text {mid }}$ is the position of the pedestal center (in general, these parameters may differ for the density and temperature profiles) in units of the normalized poloidal flux $\bar{\psi}$. The coefficients $a_{n}$, $a_{T}$ are determined by the specified values of the density $n_{\text {ped }}$ and temperature $T_{\text {ped }}$ at the top of the pedestal at $\psi_{\text {ped }}=\psi_{\text {mid }}-\Delta / 2$ and the values on the separatrix $n_{\text {sep }}, T_{\text {sep }}$. In the EPED1 model tested in experiments in the DIII-D tokamak, the width of the pedestal depends on its height in accordance with the following scaling:

$$
\Delta=0.076 \beta_{\mathrm{p}, \mathrm{ped}}^{1 / 2},
$$

where $\beta_{p, p e d}$ is the value of the poloidal beta at the top of the pedestal $\psi_{\text {ped }}$ for the profiles (1); $\beta_{\mathrm{p}, \text { ped }}=$ $2 \mu_{0} p_{\mathrm{ped}} / B_{\mathrm{p}, \mathrm{sx}}^{2}, B_{\mathrm{p}, \mathrm{sx}}^{2}=\left(\mu_{0} I_{\mathrm{p}} / L_{\mathrm{p}}\right)^{2}, B_{\mathrm{p}, \mathrm{sx}}$ is the averaged poloidal field on the separatrix, $L_{\mathrm{p}}$ is the perimeter of the separatrix. In turn, the maximum values of $\beta_{p, p e d}$ for stability of the peeling-ballooning modes depend on the width of the pedestal $\Delta$ as follows: $\beta_{\mathrm{p}, \mathrm{ped}} \sim \Delta^{3 / 4}$, which corresponds to a decrease in the limit value of the normalized pressure gradient $\alpha$ with increasing pedestal width. In [7], the scaling of the limiting $\beta_{\mathrm{p} \text {,ped }}$ from the width is refined for a wider class of profiles with an arbitrary position of the pedestal $\psi_{\text {mid }}$ $\left(\psi_{\text {mid }}>1-\Delta / 2\right.$ corresponds to the shift of the pedestal to the separatrix and large values of the pressure gradient at the boundary) using the depth of the pedestal $D=1-\psi_{\text {ped }}$; in this case it is possible to use a definition of the position of the pedestal top $\psi_{\text {ped }}$ independent of the pedestal profile: $p^{\prime}\left(\psi_{\text {ped }}\right)=(1-$ $\left.\tanh ^{2}(1)\right) p_{\max }^{\prime} \approx 0.42 p_{\max }^{\prime}$, where the maximum is taken along the pedestal, as for the pressure profile fitted by a hyperbolic tangent: for profiles (1) $D \approx \Delta$. This scaling is further generalized taking the dependence of the stability limit on the normalized current $I_{\mathrm{N}}$ into account [8]

$$
\beta_{\mathrm{p}, \mathrm{ped}}=C D^{3 / 4} / I_{\mathrm{N}}^{1 / 3},
$$

where the coefficient $C \approx 3$ for a plasma with a cross-sectional geometry similar to the ITER. Together with (2), for the depth of the pedestal $D$ close to $\Delta$ for the EPED1 profiles [7], this gives $\beta_{\mathrm{p}, \mathrm{ped}}=3^{1.6}(0.076)^{1.2} / I_{\mathrm{N}}^{8 / 15}$, which, taking into account the geometry of the plasma in the ITER $\left(L_{\mathrm{p}} / a=18.2 / 2\right)$ and with the approximate replacement of $8 / 15 \approx 0.5$, leads to a convenient expression for the limiting pressure on the pedestal

$$
p_{\text {ped }}[\mathrm{kPa}]=2 I_{\mathrm{p}}[\mathrm{MA}]^{1.5} B_{0}[\mathrm{~T}]^{0.5} / a[\mathrm{~m}]^{1.5} \text {. }
$$

For the value of the normalized beta on the pedestal $\beta_{\mathrm{N}, \text { ped }}=\left(2 \mu_{0} p_{\text {ped }} / B_{0}^{2}\right) / I_{\mathrm{N}}$, this is equivalent to

$$
\beta_{\mathrm{N}, \text { ped }}[\%]=0.5 I_{\mathrm{N}}^{0.5}
$$

for the width of the pedestal in units of the normalized poloidal flux

$$
\Delta \approx D=0.04 I_{\mathrm{N}}^{-0.25} \text {. }
$$

To obtain equilibria consistent with the pedestal model for a given width $\Delta$, which determines $\beta_{\mathrm{p} \text {,ped }}$ in accordance with (2), it is sufficient to set the plasma density at the top of the pedestal and at the separatrix, and to select the temperature according to $p_{\text {ped }}$, provided that the temperature at the plasma boundary is low: the standard value $T_{\text {sep }}=75 \mathrm{eV}$. The consistent equilibrium is determined iteratively for a given vacuum magnetic field and the value $B_{\mathrm{p}, \mathrm{sx}}$, which varies due to the current density in the pedestal. Figure 4 shows the pedestal profiles for $\Delta=0.03$ for different density distributions. It is important to note that at a lower and more peaked density, the bootstrap current is higher, which leads to a nonmonotonic safety factor profile $q$ in the pedestal. In the case of low density, this leads to a shear reversal even with a flat density profile. 
(a)
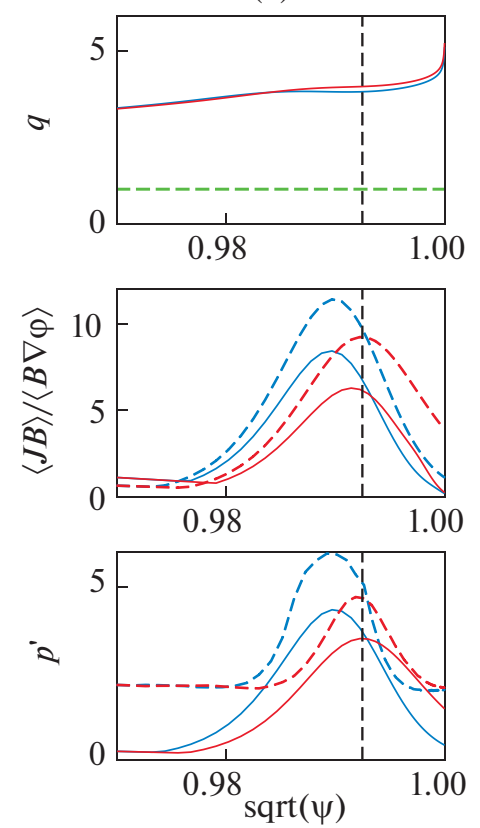

(b)
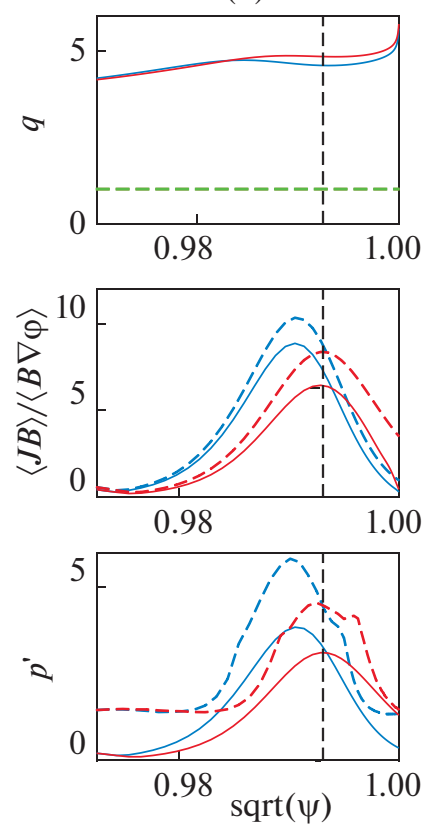

Fig. 4. Pedestal profiles for the peaked $\left(n_{\text {sep }} / n_{\text {ped }}=0.25\right.$, blue curve $)$ and flat $\left(n_{\text {sep }} / n_{\text {ped }}=1\right.$, red curve) density distribution in the pedestal, $\Delta=0.03, \beta_{\mathrm{p} \text {,ped }}=0.156, B_{0}=8 \mathrm{~T}$ : equilibrium with Fig. $1 \mathrm{c}$, (a) $n_{\text {ped }}=15 \times 10^{19} \mathrm{~m}^{-3}, T_{\text {ped }}=1.68$ and $1.60 \mathrm{keV}$, $p_{\text {ped }}=81$ and $77 \mathrm{kPa}, I_{\mathrm{p}}=4.87$ and $4.76 \mathrm{MA}$ and (b) equilibrium with Fig. $3 \mathrm{a}, n_{\text {ped }}=5 \times 10^{19} \mathrm{~m}^{-3}, T_{\text {ped }}=3.46$ and $3.35 \mathrm{keV}$, $p_{\text {ped }}=55$ and $52 \mathrm{kPa}, I_{\mathrm{p}}=4.0$ and $3.95 \mathrm{MA}$. The dashed curves show the density of the collisionless bootstrap current and the pressure gradient limiting for ballooning modes. Vertical dashed lines show the position of the center of the pedestal $\sqrt{1-\Delta / 2}=0.9925$.

\section{MAXIMUM STABILITY PARAMETERS OF THE PEDESTAL AND TAKING INTO ACCOUNT THE DIAMAGNETIC STABILIZATION}

\subsection{Maximum Height of the Pedestal and the Stability Diagram}

In the framework of the EPED1 model, it is possible to construct a sequence of equilibria with an increase in pressure at the top of the pedestal and a corresponding increase in the width of the pedestal, and then determine the limit against the stability of the peeling-ballooning modes. Another way to study the stability of the pedestal is to calculate stability diagrams [6]. In this case, the profiles of the pressure gradient and the parallel current density can be proportionally changed with a fixed width of the pedestal [8]. For each mode with a given toroidal wave number $n$, the stability boundaries are found in the parametric plane $\left(\alpha, J_{\|} /\langle J\rangle\right)$, where the normalized pressure gradient at the center of the pedestal $\psi_{\text {mid }}$ is defined as in [9]

$$
\alpha=\frac{2 \mu_{0}}{4 \pi^{2}} \frac{d p}{d \psi} \frac{d V_{\psi}}{d \psi} \sqrt{\frac{V_{\psi}}{2 \pi^{2} R}},
$$

and the parallel current density $J_{\|}=\langle\mathbf{j} \cdot \mathbf{B}\rangle_{\psi} /\langle\mathbf{B} \mid\rangle_{\psi}$ (averaging over the volume between the magnetic surfaces) is normalized by the average cross-sectional density of the total plasma current $\langle J\rangle=I_{\mathrm{p}} / S_{\mathrm{p}}$.

\subsection{Diamagnetic Stabilization}

To estimate the effect of diamagnetic stabilization, which occurs in the simplest model at $\gamma<\omega_{*} / 2$ [10], the diamagnetic frequency given by the following expression [11] is: $\omega_{*}=\omega_{* p i}=\left(n / n_{i} e_{i}\right)\left(d p_{i} / d \psi\right)$, where $\psi$ is the poloidal flux, $p_{i}, n_{i}, e_{i}$ are the pressure, density, and charge of ions, and $n$ is the toroidal wave number. It is convenient to express $\omega_{*}$ in terms of the ion-cyclotron frequency $\omega_{B i}=e_{i} B_{0} / m_{i}$ and the Alfvén frequency $\omega_{\mathrm{A}}=B_{0} /\left(R \sqrt{\mu_{0} \rho}\right)$ :

$$
\omega_{*}=n \frac{\omega_{\mathrm{A}}}{\omega_{B i}} \omega_{\mathrm{A}} \frac{R^{2}}{B_{0}} \mu_{0} \frac{d p_{i}}{d \psi},
$$

where $m_{i}$ is the mass of the ion, $B_{0}$ is the vacuum magnetic field, $R$ is the major radius of the plasma, and $\rho$ is the mass density on the magnetic axis. The frequency of the diamagnetic drift of ions is calculated in 


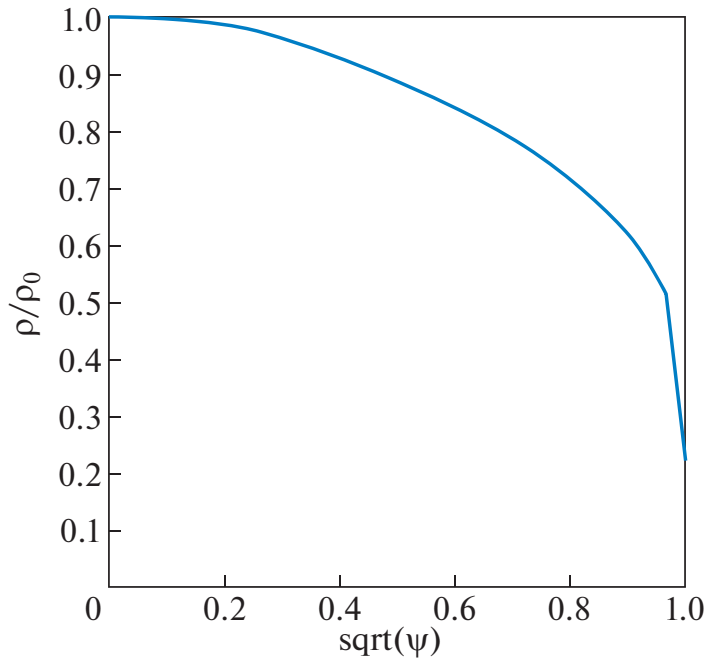

Fig. 5. Normalized plasma mass density for calculating growth rates.

the middle of the plasma pressure pedestal, assuming that $p_{i}^{\prime}=p^{\prime} / 2$. Diamagnetic stabilization is especially effective for modes with a high toroidal wave number, to which the value of the diamagnetic frequency is proportional. To calculate the growth rate, the mass density profile corresponding to ASTRA-code modeling is used (Fig. 5).
To calculate the stability diagrams, the equilibria from Fig. 4 were used for a fixed width of the pedestal $\Delta=0.03$ (Figs. 6 and 7). The initial equilibria feature the same poloidal beta $\beta_{\mathrm{p}, \mathrm{ped}}=(\Delta / 0.076)^{2}=0.156$. In this case, the coefficient $C$ from scaling (5) for the height of the pedestal is lower than the standard limit $C=3$ for an ITER-like plasma: $C=2.2-2.1$. This corresponds to the calculated stability of the initial equilibria from Fig. 6 (peaked pressure profile and relatively high $\beta_{\mathrm{p}}=0.81$ ), but contradicts the stability of the equilibria from Fig. 7 (nonmonotonic $q, \beta_{\mathrm{p}}=$ $0.56)$. In the latter case, taking into account diamagnetic stabilization only gives stability: for deuterium plasma in TRT with a density on the magnetic axis $n_{i}=$ $2 \times 10^{20}\left(10^{20}\right) \mathrm{m}^{-3}$ and $R=2.15 \mathrm{~m}$, we have $\omega_{\mathrm{A}} / \omega_{B i}=$ 0.0106 (0.015) for Fig. 6 (Fig. 7).

Tables 1 and 2 show the maximum parameters of the pedestal according to the EPED1 method, i.e., for a sequence of equilibria with profiles (1) at the width of the pedestal (2): the density on the pedestal $n_{\text {ped }}=$ $15 \times 10^{19}$ and $5 \times 10^{19} \mathrm{~m}^{-3}$. The coefficient $C=$ $\beta_{\mathrm{p}, \mathrm{ped}}\left(\psi_{\mathrm{ped}}\right) I_{\mathrm{N}}^{1 / 3} / \Delta^{3 / 4}, \psi_{\text {ped }}=1-\Delta$ is calculated like scaling (5) for the limit value of the width of the pedestal $\Delta$. It should be noted that the stability limits are reached in equilibria with a reversed shear in the pedestal. In this case, it is possible to destabilize the ideal modes when the resonant magnetic surface (a)

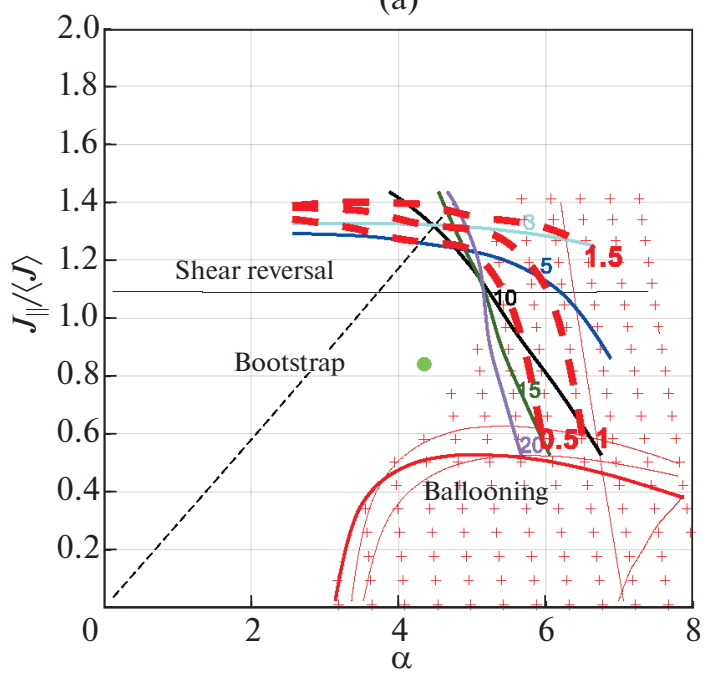

(b)

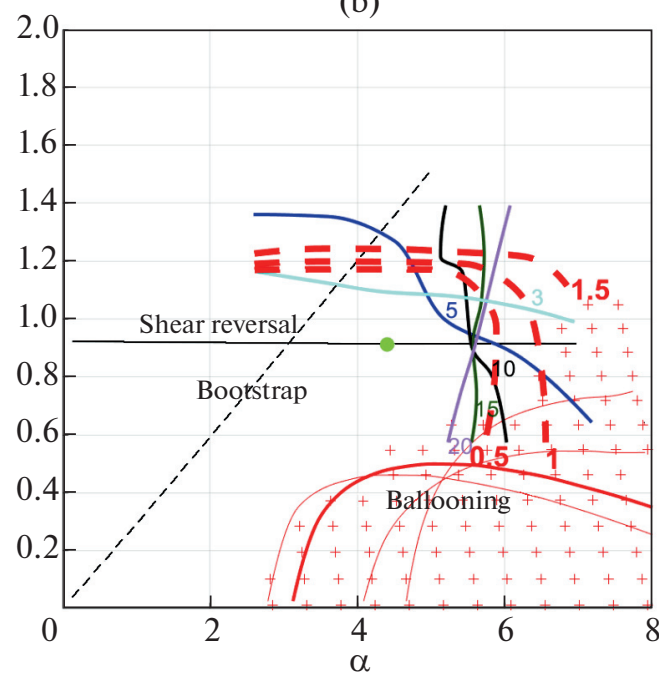

Fig. 6. Comparison of stability diagrams in the plane $\left(\alpha, J_{\|} /\langle J\rangle\right)$ for equilibria in Fig. 4a: flat density (a); peaked density (b), in this case, the maximum current density and the normalized pressure gradient are approximately 1.3 and 1.2 times greater than the corresponding values at the center of the pedestal $\psi_{\text {mid }}$, respectively. The crosses show the region of instability in small-scale ballooning modes, the solid red lines are the boundaries of their stability on several magnetic surfaces in the pedestal. The shear reversal (nonmonotonic $q$ ) occurs above a thin solid line. The dashed thin line shows the current density corresponding to the collisionless bootstrap current. The bold lines correspond to the stability boundaries for peeling-ballooning modes with numbers $n=5-20$ (toroidal wave numbers are written). The light-colored lines show the stability boundaries for global modes with $n=3$. The circle at the center of the diagram corresponds to the parameters of the pedestal for the initial equilibrium. The thick dashed lines correspond to the level lines of the growth rate normalized to the diamagnetic frequency $\gamma /\left(\omega_{*} / 2\right)=0.5,1.0,1.5$. 
(a)

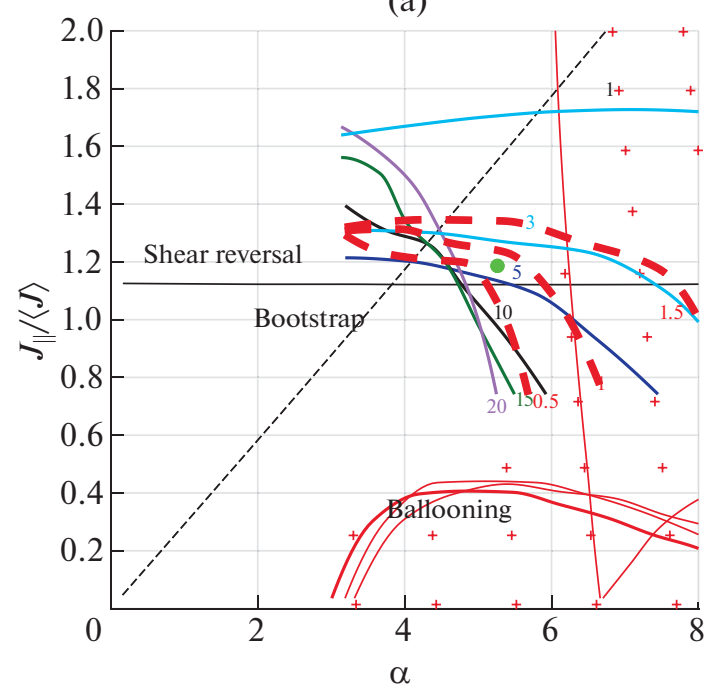

(b)

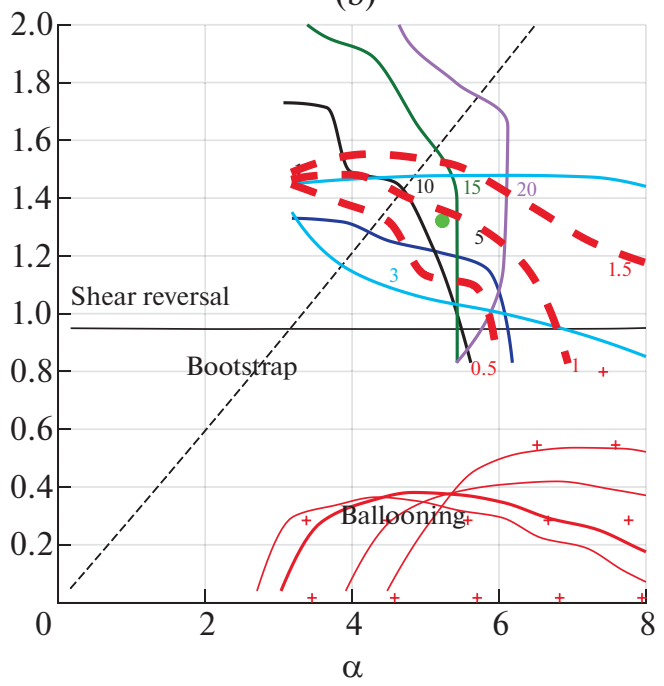

Fig. 7. Comparison of the stability diagrams in the parametric plane $\left(\alpha, J_{\|} /\langle J\rangle\right)$ for the equilibria in Fig. $4 b$ : (a) flat density and (b) peaked density. The light-colored lines show the stability boundaries for global modes with $n=1$ and 3 .

(a)

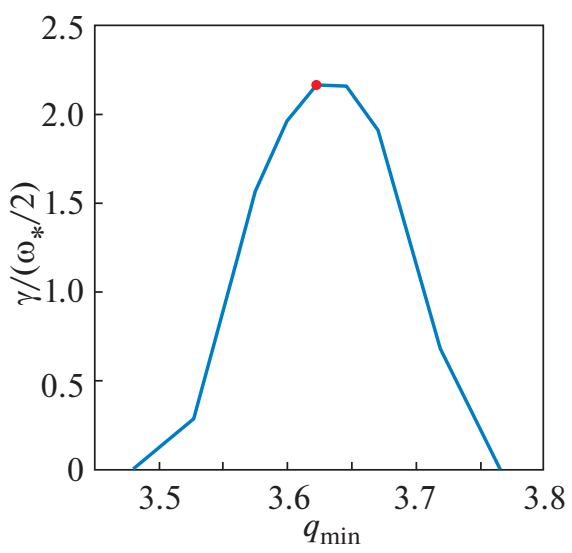

(b)

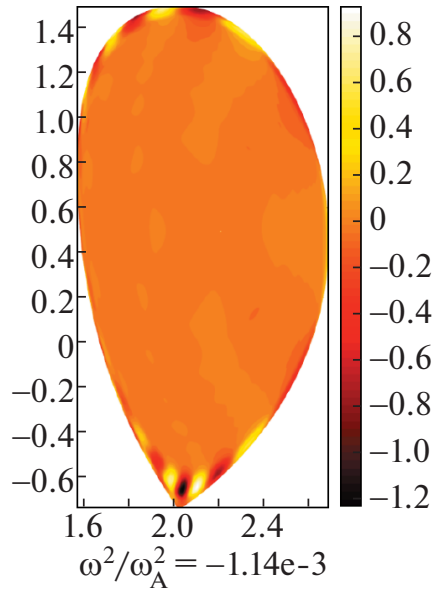

(c)

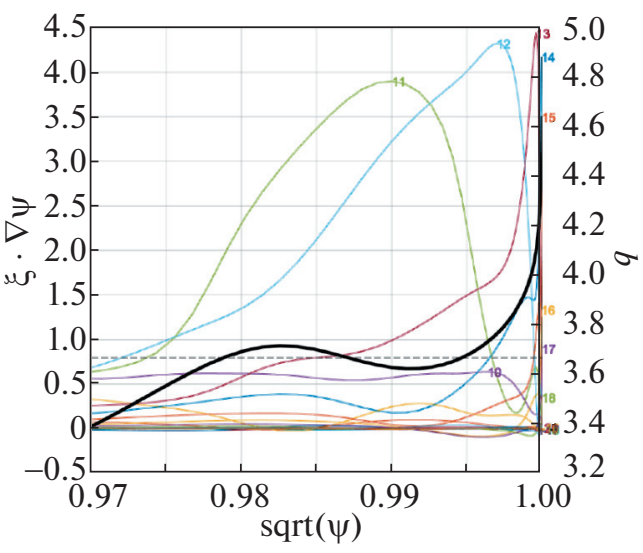

Fig. 8. Dependence of the growth rate normalized to the diamagnetic frequency; (a) $\gamma /\left(\omega_{*} / 2\right)>1$ corresponds to instability and (b, c) the structure of the unstable mode $n=3$ with a free boundary: the lines of the level of the displacement normal to the magnetic surfaces and the harmonics of radial displacement $\xi \cdot \nabla \psi$ (arbitrary units). The bold line shows the $q$ profile, the dashed line is $q=11 / 3$, and $q_{\mathrm{min}}=3.62$. The width of the pedestal $\Delta=0.035$.

approaches the region of a small shear and a large pressure gradient (infernal modes [5]), as well as their resistive analogues, including double tearing modes. Thus, the achievement of current density in the pedestal, which leads to reversal of the shear even before destabilization of the peeling-ballooning modes, can be interpreted as another limit of the pedestal stability. On the other hand, the existence of three-dimensional equilibrium configurations with a large contribution of the harmonic $n=1$ at the boundary and corresponding to the nonlinear saturation of kink modes with a significant bootstrap current (quiescent $\mathrm{H}$-mode $(\mathrm{QH})$ ) can be associated with a small or reversed shear in the pedestal [12].

The last two rows in Table 1 show that for this series of equilibrium configurations, the $n=3$ mode is destabilized with a sharp increase in the increment, so that diamagnetic stabilization is ineffective. This is illustrated in Fig. 8, which shows the dependence of the growth rate normalized by the diamagnetic frequency at the local minimum value of $q_{\min }$, when the 
Table 1. Maximum values of the pedestal width, the number of the most unstable mode and the coefficient in the scaling of the pedestal height for equilibria with a peaked pressure profile and monotonic $q, n_{\text {ped }}=15 \times 10^{19} \mathrm{~m}^{-3}$ for different density profiles in the pedestal with and without taking into account the diamagnetic stabilization

\begin{tabular}{c|c|c|c}
\hline & $\Delta_{\text {lim }}$ & $n$ & $C$ \\
\hline$n_{\text {sep }} / n_{\text {ped }}=1$ & 0.0341 & 15 & 2.54 \\
diamagn. stabil. & 0.0362 & 10 & 2.82 \\
$n_{\text {sep }} / n_{\text {ped }}=0.25$ & 0.0329 & 3 & 2.48 \\
diamagn. stabil. & 0.0329 & 3 & 2.48 \\
\hline
\end{tabular}

Table 2. Maximum values of the pedestal width, the number of the most unstable mode and the coefficient in the scaling of the pedestal height for equilibria with a flat pressure profile and nonmonotonic $q, n_{\text {ped }}=5 \times 10^{19} \mathrm{~m}^{-3}$ for different density profiles in the pedestal with and without taking into account the diamagnetic stabilization

\begin{tabular}{c|c|r|c}
\hline & $\Delta_{\lim }$ & $n$ & $C$ \\
\hline$n_{\text {sep }} / n_{\text {ped }}=1$ & 0.0281 & 15 & 1.85 \\
diamagn. stabil. & 0.0307 & 5 & 2.09 \\
$n_{\text {sep }} / n_{\text {ped }}=0.25$ & 0.0276 & 15 & 1.85 \\
diamagn. stabil. & 0.0302 & 3 & 2.09 \\
\hline
\end{tabular}

plasma current changes, and demonstrates the structure of the mode. The maximum of the growth rate is attained when the resonant surface $m / n=11 / 3$ approaches $q_{\min }$, i.e., just for the parameters of the EPED1 equilibrium with the pedestal width $\Delta=0.035$.

\section{CONCLUSIONS}

Calculations of the ideal MHD stability of the TRT-tokamak plasma, taking into account the pedestal, indicate operating limits of the installation with normalized $\beta_{\mathrm{N}} \approx 2$, which are lower than those for ITER-like plasma: this is due to a lower TRT-plasma shape factor. For the same reason, as well as due to reversal of the shear in the pedestal at lower bootstrapcurrent values, the height of the pedestal is limited by scaling (3) with the coefficient $C=2-2.5$. Taking into account the dependence $C^{1.6}$ for the height of the pedestal in equilibria with the width of the pedestal (2), it is necessary to correct the scalings (4) and (5): $(2 / 3)^{1.6} \approx 0.5,(2.5 / 3)^{1.6} \approx 0.75$. The low shape factor is partially compensated by a large Shafranov shift for equilibria with a fixed pressure profile and large $\beta_{\mathrm{p}}$. Increasing the triangularity of the plasma cross section is the most effective way to increase the maximum beta and the height of the pedestal. The TRT poloidal coil system allows the formation of plasma configurations with different triangularity [13], which, along with the control of profiles using additional heating and current-drive methods, will allow optimization of the plasma parameters necessary to achieve the expected characteristics of the installation. Under the assumption of a high plasma-confinement mode $(\mathrm{H}$-mode $)$ and in the presence of the type-I ELMs, the pulse load on the TRT divertor plates can be estimated as $\Delta W_{\mathrm{ELM}} / W_{\text {ped }} \sim 5-10 \%$, where $W_{\text {ped }}=3 / 2 n_{e, \text { ped }} \times$ $\left(T_{e, \text { ped }}+T_{i, \text { ped }}\right) V_{\text {plasma }}[14]$. At a pressure on the pedestal of $80 \mathrm{kPa}$ and a plasma volume of $V_{\text {plasma }}=24.2 \mathrm{~m}^{3}$, this estimate gives $\Delta W_{\mathrm{ELM}}=150-300 \mathrm{~kJ}$.

\section{FUNDING}

The work was carried out with financial support of State Atomic Energy Corporation Rosatom under agreement no. 313/1671-D from September 5, 2019.

\section{OPEN ACCESS}

This article is licensed under a Creative Commons Attribution 4.0 International License, which permits use, sharing, adaptation, distribution and reproduction in any medium or format, as long as you give appropriate credit to the original author(s) and the source, provide a link to the Creative Commons licence, and indicate if changes were made. Theimages or other third party material in this article are included in thearticle's Creative Commons licence, unless indicated otherwise in a credit line to the material. If material is not included in the article's Creative Commons licence and your intended use is not permitted by statutory regulation or exceeds the permitted use, you will need to obtain permission directly from the copyright holder. To view a copy of this licence, visit http://creativecommons.org/licenses/by/4.0/.

\section{REFERENCES}

1. A. V. Krasilnikov, S. V. Konovalov, E. N. Bondarchuk, I. V. Mazul, I. Yu. Rodin, A. B. Mineev, E. G. Kuz'min, A. A. Kavin, D. A. Karpov, V. M. Leonov, R. R. Khayrutdinov, A. S. Kukushkin, D. V. Portnov, A. A. Ivanov, Yu. I. Belchenko, et al., Plasma Phys. Rep. 47, 1092 (2021).

2. O. Sauter, C. Angioni, and Y. R. Lin-Liu, Phys. Plasmas 6, 2834 (1999).

3. O. Sauter, C. Angioni, and Y. R. Lin-Liu, Phys. Plasmas 9, 5140 (2002).

4. L. Degtyarev, A. Martynov, S. Medvedev, F. Troyon, L. Villard, and R. Gruber, Comput. Phys. Commun. 103, 10 (1997).

5. A. R. Polevoi, A. A. Ivanov, S. Yu. Medvedev, G. T. A. Huijsmans, S. H. Kim, A. Loarte, E. Fable, and A. Y. Kuyanov, Nucl. Fusion 60, 096024 (2020).

6. P. B. Snyder, R. J. Groebner, A. W. Leonard, T. H. Osborne, and H. R. Wilson, Phys. Plasmas 16, 056118 (2009). 
7. S. Yu. Medvedev, A. A. Ivanov, A. A. Martynov, Yu. Yu. Poshekhonov, R. Behn, Y. R. Martin, J.-M. Moret, F. Piras, A. Pitzschke, A. Poshelon, O. Sauter, and L. Villard, Contrib. Plasma Phys. 50, 324 (2010).

8. S. Yu. Medvedev, A. A. Ivanov, A. A. Martynov, Yu. Yu. Poshekhonov, S. V. Konovalov, and A. R. Polevoi, Plasma Phys. Rep. 42, 472 (2016).

9. R. J. Groebner and T. H. Osborne, Phys. Plasmas 5, 1800 (1998).

10. G. T. A. Huysmans, S. E. Sharapov, A. B. Mikhailovskii, and W. Kerner, Phys. Plasmas 8, 4292 (2001).
11. R. J. Hastie, P. J. Catto, and J. J. Ramos, Phys. Plasmas 7, 4561 (2000).

12. D. Brunetti, J. P. Graves, E. Lazzaro, A. Mariani, S. Nowak, W. A. Cooper, and C. Wahlberg, Phys. Rev. Lett. 122, 155003 (2019).

13. E. N. Bondarchuk, A. A. Kavin, A. B. Mineev, S. V. Konovalov, V. E. Lukash, and R. R. Khayrutdinov, Plasma Phys. Rep. 47 (2021) (in press).

14. A. Loarte, G. Saibene, R. Sartori, D. Campbell, M. Becoulet, L. Horton, T. Eich, A. Herrmann, G. Matthews, N. Asakura, A. Chankin, A. Leonard, G. Porter, G. Federici, G. Janeschitz, et al., Plasma Phys. Control. Fusion 45, 1549 (2003). 